\title{
MÁQUINA CLASIFICADORA DE FLORES: DISEÑO Y CONSTRUCCIÓN
}

\section{FLOWERS CLASSIFICATION MACHINE: DESIGN AND CONSTRUCTION}

\author{
Ing. Freddy Martínez, Ing. Hernandez David, PhD. Pedro F. Cárdenas Herrera. \\ Universidad Nacional de Colombia sede Bogotá, Facultad de Ingeniería. \\ Departamento de Ingeniería Mecánica y Mecatrónica. \\ Ciudad Universitaria, Cr 30 \#45-14, Bogotá. \\ E-mail: \{fhmartinezq, daahernandezca, pfcardenash\}@unal.edu.co
}

\begin{abstract}
Resumen: Las máquinas clasificadoras tienen como objetivo principal optimizar el proceso de clasificación de cualquiera que sea el producto con el que trabajan, es decir, mejorar los resultados, disminuir los gastos y aumentar la velocidad del proceso. Este documento presenta el proceso de diseño y construcción de una máquina de clasificación de flores, la cual hace uso de técnicas de visión de máquina e inteligencia artificial. Esta máquina fue proyectada para desempeñarse bajo las condiciones ambientales de las salas de pos-cosecha en los invernaderos de la sabana de Bogotá.
\end{abstract}

Palabras clave: Automatización de procesos agro-industriales, clasificación de flores, máquina clasificadora, visión artificial.

\begin{abstract}
Sorting machines are aimed to optimize the sorting process whatever the product they work with, that is, improve performance, reduce costs and speed up the process. This paper presents the process of designing and building a flower sorting machine, which uses machine vision techniques and artificial intelligence. This machine was designed to function under current environmental conditions of post-harvest rooms of the greenhouses of the savannah of Bogota.
\end{abstract}

Keywords: Agroindustrial processes automation, flowers classification, sorting machine, machine vision.

\section{INTRODUCCIÓN}

En el negocio de cultivo de flores de exportación, un proceso muy importante es la clasificación. La clasificación de la flor depende del mercado al que se dirige, de acuerdo con la vida media de la rosa ésta puede o no llegar en condiciones adecuadas al destino de exportación. El proceso de clasificación requiere de mucha atención (AFIF, 2010) y necesita de personal con amplia experiencia, el cual es escaso en el mercado laboral de la zona. Por lo cual se presentan dos inconvenientes: el primero los costos elevados de mano de obra; el segundo un cuello de botella debido al número reducido de personas capacitadas en la etapa de clasificación (Monroy, 2002).

Este proceso ha sido profundamente explorado usando algoritmos de aprendizaje de máquina como SVM (Zawbaa, 2014) o redes neuronales (Siraj, 2014). Además, el uso de visión de máquina se ha probado útil a la hora de realizar tareas de clasificación durante diferentes procesos de producción [Pardo, 2013], incluyendo clasificación en tiempo real para el sector agrícola (Katupitiya, 2007) como es resumido por Sistler (Sistler), quien muestra que se ha utilizado para la clasificación de la calidad de granos de arroz y de cangrejos en producción masiva. 
Actualmente los procesos agrícolas en Colombia se realizan en su gran mayoría de forma manual, a falta de soluciones tecnológicas que permitan un aumento en la producción que sea atractivo para los productores.

En el presente trabajo se propone el diseño y construcción de una máquina clasificadora de flores a partir de visión artificial, el objetivo de la máquina es mejorar el proceso de producción de rosas, agilizando específicamente la etapa de clasificación de las mismas, dentro de las diversas etapas que implica el proceso de producción. La máquina debe ser capaz de homogenizar el material entregado al reducir o eliminar los errores cometidos por la intervención humana, como es el error de paralaje, errores por fatiga o por falta de experiencia del operador. La clasificación se hace considerando parámetros morfológicos de las flores, parámetros que permiten el análisis y comparación de estructuras de seres vivos (Ferrin, 2014), como son: tamaño del botón lateral y frontal, número de pétalos y el grado de apertura de la rosa.

\section{MÉTODO Y DESARROLLO DE LA IDEA}

En el desarrollo de la idea se contó con el apoyo de un finca floricultura que expreso su necesidad de mejorar ese proceso en particular. Con ayuda de ellos se definieron algunas cualidades que debía tener la máquina, como: volumen, capacidad de procesamiento, costo, disminución de la cantidad de flores rechazadas por mal manejo de producto, etc.

En seguida se evaluaron los parámetros de ingeniería necesarios por el dispositivo para un correcto funcionamiento, algunos de los que en nuestro caso se definieron como parámetros clave son: velocidad de procesamiento, volumen, escalabilidad y uso de elementos estándar.

Una vez definidas las prioridades sobre los parámetros de diseño de la maquina se elabora una matriz QFD (Despliegue de la función de calidad), donde se evalúan los valores más importantes a tener en cuenta dentro del desarrollo del prototipo y las relaciones que deben existir entre ellos. A continuación se muestra un diagrama de caja gris (Fig. 1), donde se evidencia la interacción entre las principales partes de la máquina.

El diseño, como se evidencia en la figura 1, se dividió en tres secciones principales independientes, de modo que fuese posible desarrollar cada una de ellas de forma simultánea, teniendo como tarea final su acoplamiento. Estas subdivisiones fueron: el software para el reconocimiento y clasificación, la estructura mecánica y la electrónica para las comunicaciones y el control del sistema.

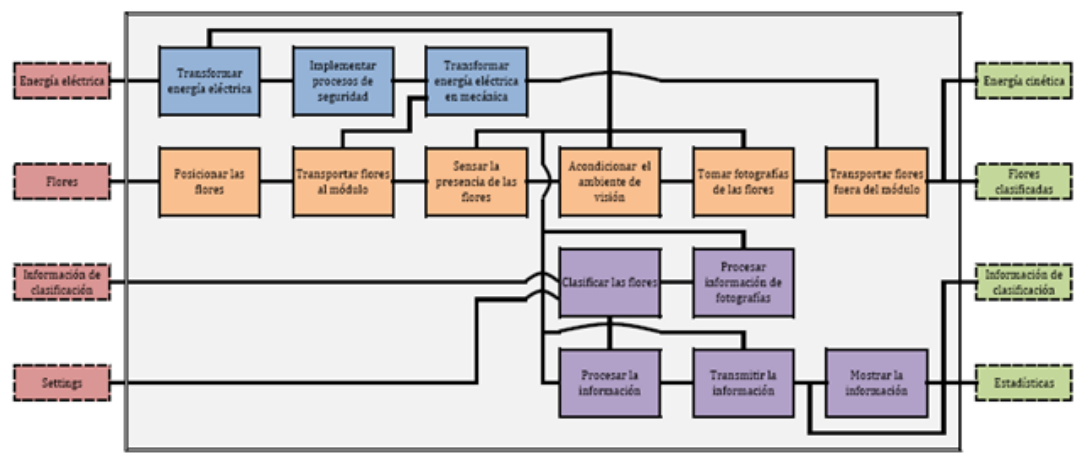

Fig. 1. Caja gris del prototipo.

\subsection{Diseño mecánico}

Para el desarrollo del sistema mecánico, concebido como el medio de transporte de las rosas, se realizaron dinámicas como lluvias de ideas acompañadas de bocetos sobre el funcionamiento general de la máquina.
Una vez planteado el diseño general se llevó a cabo una descomposición funcional de la máquina, evaluando la solución más práctica de cada una de las funciones, llevando la evaluación hacia la selección de piezas individuales. Una vez en este punto, se comparan y seleccionan las mejores opciones para determinadas funciones según los requerimientos especificados. 
En primera instancia se lleva a cabo la comparación de los elementos individuales a través de una matriz de selección, filtrando los elementos que mejores características presenten, después se procede a generar combinaciones de elementos, acomodándolos en diferentes configuraciones y a evaluarlos en conjunto, para llegar a una idea global de la máquina con todas sus partes.

A partir de esta idea, se empieza a desarrollar los esquemas, planos y modelos en software especializado con el objetivo de visualizar el producto, a la vez que se evalúan todos los requerimientos e implicaciones de diversa índole que acarrea el uso de cada pieza o conjunto; de este modo se da lugar a un proceso iterativo de diseño, dimensionamiento y ajuste de todas las partes, a través del cual se va adecuando y ensamblando todo el sistema mecánico hasta llegar a su versión definitiva.

\subsection{Desarrollo del software}

El software del prototipo fue desarrollado sobre una plataforma Linux que facilita el manejo de un mayor número de interfaces diferentes.

Se configuraron los drivers necesarios para el manejo de las cámaras BASLER SC640 - 70FPS, en particular se destaca el uso del software coriander como plataforma de prueba de la capacidad de adquisición de las dos cámaras.

Las cámaras basler utilizan la interfaz de comunicación FireWire 800 (IEEE 1394b). Para la instalación de coriander basta con el siguiente comando: apt-get install coriander.

Una vez verificada la conexión de las cámaras, se utilizan para construir una base de datos que permita el entrenamiento de un clasificador de rosas explicado a continuación.

La interfaz de control y clasificación fue desarrollada en Qt (Ver fig. 3) sobre C++, el cual permite la interacción de un usuario sin mayor entrenamiento con la máquina. Dentro de esta interfaz además de los controles de manejo de la máquina, se muestran estadísticas de clasificación y una muestra de las rosas siendo clasificadas en el interior.

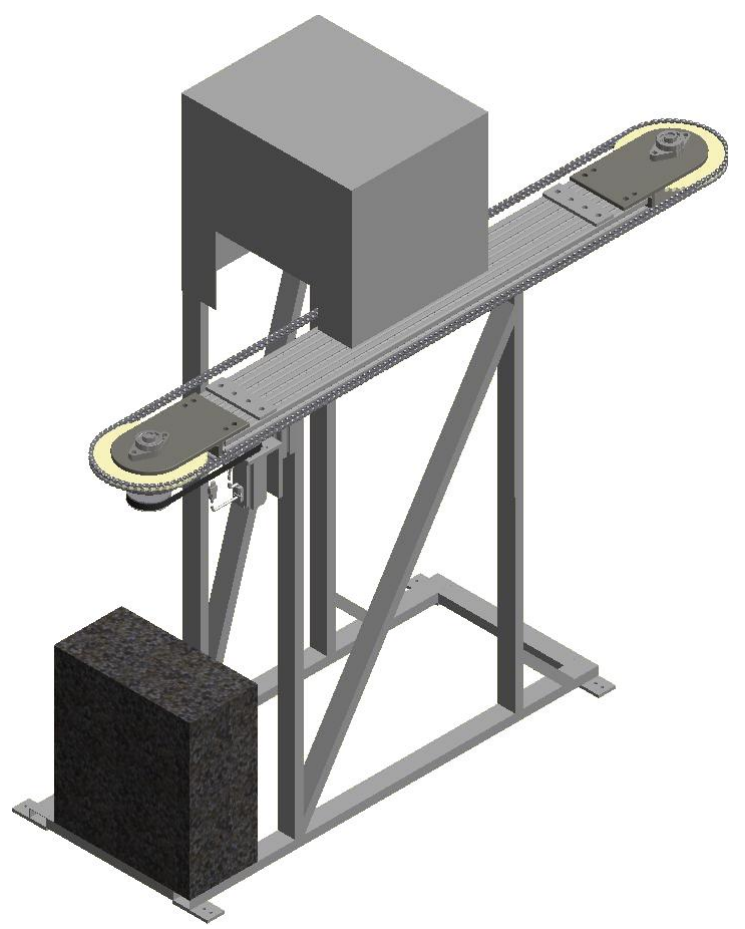

Fig. 2. Modelo desarrollado en el software CAD Inventor.

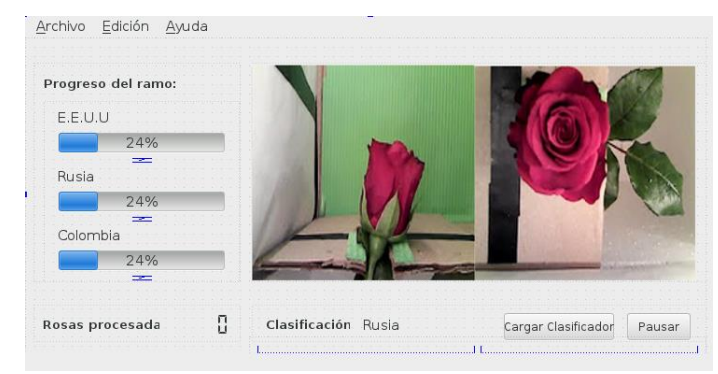

Fig. 3. Interfaz gráfica creada en QtCreator.

El software utiliza Open $C V$ para el pretratamiento y la extracción de características, además de los drivers de control de la interfaz IEEE 1394 para C++. Además se comunica usando el puerto serial del computador por medio de la librería de QtSerialPort.

En la librería de comunicación serial se instala como se ve en la página web QtSerialPort y Opencv se instala como se ve en la página web Install Open $C V$.

El diagrama de flujo de la figura Fig. 4 resume en términos generales el proceder del programa en su totalidad. 


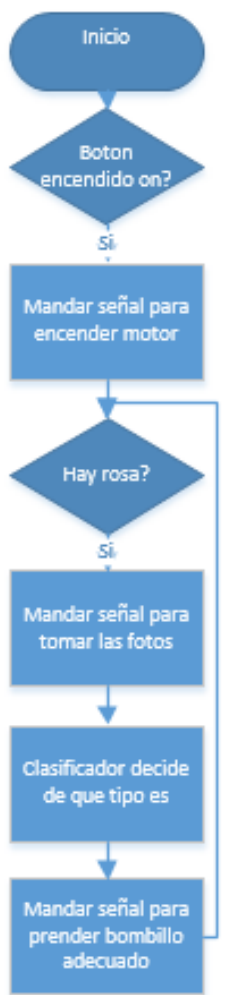

Fig. 4. Diagrama de flujo del prototipo.

Como algoritmo de clasificación de rosas se utilizó un clasificador por distancias euclidianas entre una ponderación de 3 características principales: tamaño del botón (ancho, alto) y la densidad de pétalos de la rosa. El clasificador se entrenó en base a estas características, las cuales se extrajeron de la base de datos creada a partir de muestras de las 3 clases de rosas proporcionadas por la finca floricultora, basadas en los 3 tipos de mercados para los cuales se comercializan las rosas (Estados Unidos, Rusia y Nacional).

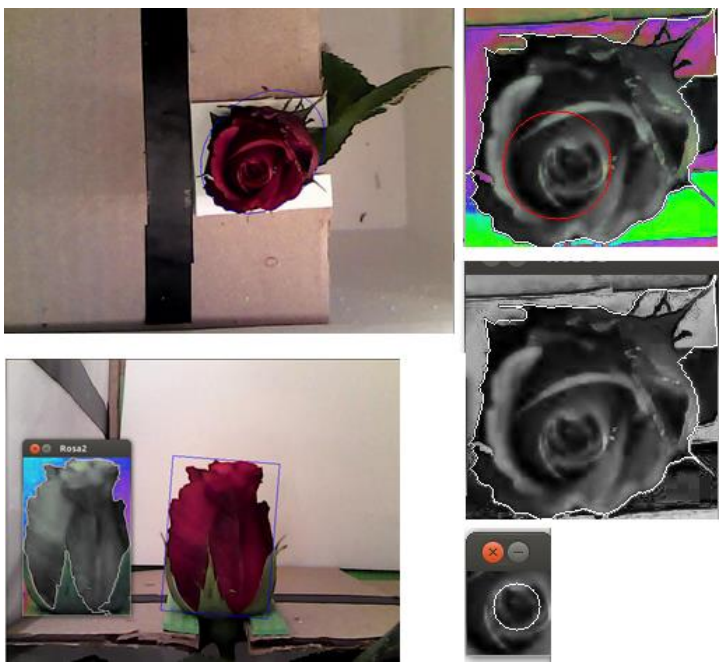

Fig. 5. Tratamiento de la imagen.

\subsection{Sistemas electrónicos}

Debido a que se determinó el uso de un servomotor como mejor fuente de potencia mecánica, fue necesario desarrollar el tablero de entrada de energía para el servopack que lo controla, el cual se observa en la Fig. 6.

La detección de presencia de la rosa para la adquisición de la imagen se lleva a cabo usando un sensor analógico, el cual envía la información a través de la tarjeta de desarrollo Arduino, a la vez que la salida de la información de clasificación también se hace a través de la misma tarjeta Arduino. Se determina hacer uso de los dispositivos empleados para la entrada de corriente del servopack para proveer energía a los bombillos pilotos (destinados para mostrar el resultado de la clasificación) y a la fuente (encargada de suministrar energía al circuito que gobierna los parámetros de movimiento del servopack). De este modo, simplemente hace falta hacer uso de relevadores de baja corriente para acoplar las salidas del Arduino con los pilotos alimentados a $220 \mathrm{~V}$.

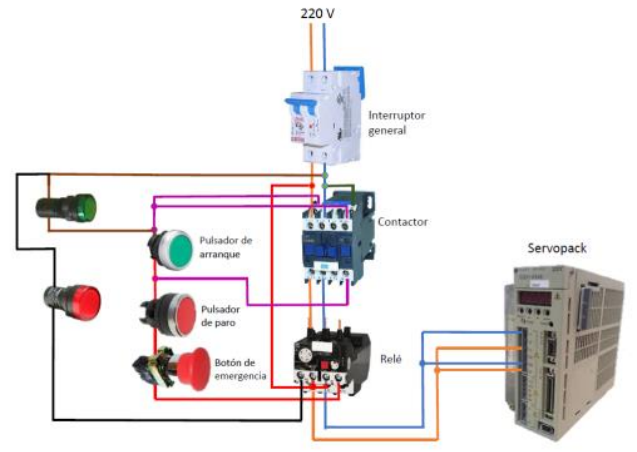

Fig. 6. Diagrama de circuito de entrada de energía de la máquina.

\section{PROCESO DE CONSTRUCCIÓN}

Una vez obtenido el diseño definitivo, se procedió a llevar a cabo su construcción: Se realizó el montaje mecánico, seguido del montaje eléctrico y el ensamble todos los sistemas (mecánico, electrónico e informático).

- En cuanto al montaje mecánico se compraron las piezas estándar y se mandaron a fabricar las piezas especiales en una empresa de manufactura local, acto seguido se procedió a ensamblar todo el sistema (ver Fig. 7). 


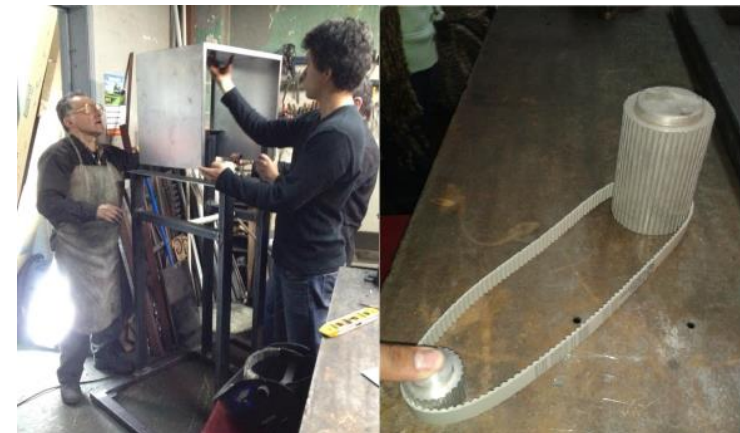

Fig. 7. Construcción del sistema mecánico.

- $\quad$ Para el montaje eléctrico, correspondiente a la entrada de potencia y el control de movimiento de la máquina se compraron los materiales y se llevaron a cabo las conexiones según el diseño (el montaje se observa en la Fig. 8).

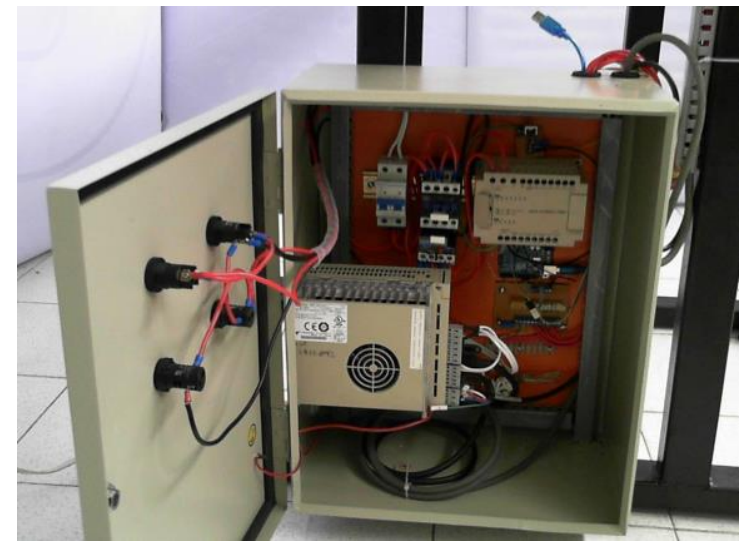

Fig. 8. Gabinete de energía.

- El ensamble de los sistemas consistió en conectar definitivamente todo y probar las comunicaciones: las imágenes captadas y enviadas por las cámaras, la iluminación, los bombillos pilotos, la señal del sensor de presencia y la velocidad del servomotor.

\section{RESULTADOS}

Terminado de montar y acoplar todos los sistemas de la máquina se procedió a realizar pruebas y ajustes en los diferentes sistemas: en la comunicación para solucionar problemas de ruido de los sensores; en el código para la recepción de datos y envío de señales para el movimiento y las salidas de la máquina. Ver Fig. 9.

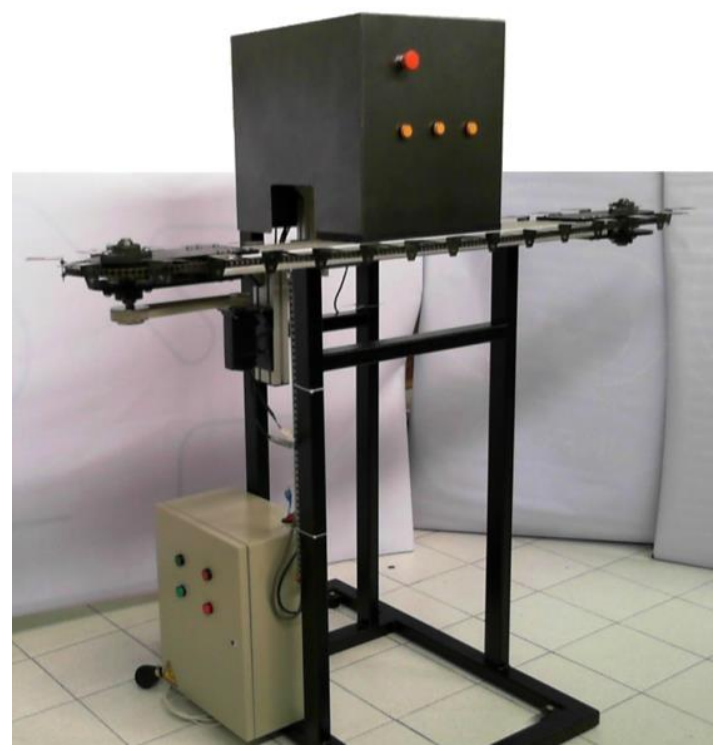

Fig. 9. Máquina clasificadora diseñada y construida.

Finalmente, la máquina dio como resultado una precisión cercana al 80\%, donde los errores más comunes se presentaban al clasificar rosas de las categorías de Estados Unidos y Rusia, tal como se ve en la Tabla 1, la cual corresponde a una muestra de los resultados entregados en una de las pruebas realizadas, donde se evidencian los resultados de clasificación de 6 especímenes diferentes, dos ejemplares de cada categoría.

Tabla 1. Resultados finales.

\begin{tabular}{cc}
\hline Mercado real & Clasificación de la máquina \\
\hline Colombia & Colombia \\
Estados Unidos & Estados Unidos \\
Rusia & Estados Unidos \\
Colombia & Colombia \\
Estados Unidos & Rusia \\
Rusia & Rusia \\
\hline
\end{tabular}

\section{RECONOCIMIENTOS}

A la Universidad Nacional de Colombia que a través de la DIB financia proyectos de semilleros.

A los ingenieros Flavio Pietro y Guillermo Cárdenas por el apoyo con dispositivos para el desarrollo del proyecto. A los estudiantes Andrea Gabriela Juyó y Francisco Alfonso, quienes fueron de gran apoyo trabajando con esmero en el desarrollo de la máquina. A William Roa (encargado del Lab. de Mecatrónica) por su apoyo en el proceso de construcción y pruebas de la máquina. A COLCIENCIAS por la Beca de doctorado de Pedro Cárdenas. 


\section{CONCLUS IONES}

Para un comportamiento adecuado del sistema es necesario que la velocidad de la banda sea constante, es importante el correcto dimensionamiento del motor para evitar problemas por la inercia de la cadena o por fricción.

Los sistemas de soporte de las rosas son funcionales, sin embargo al llegar al piñón, la aceleración angular que se produce por el movimiento a veces irregular de la banda hace que las flores se desacomoden, por tanto en versiones posteriores es necesario mejorar el sistema de soporte.

Este proyecto permite demostrar la posibilidad de mejorar las condiciones de trabajo de quienes actualmente llevan a cabo el proceso de clasificación de rosas, al alejar estas personas de posibles riesgos laborales, a la vez que permite eliminar el cuello de botella del proceso de producción de rosas.

Se demuestra además la utilidad de la visión de máquina en aplicaciones industriales y productivas.

\section{REFERENCIAS}

AFIF, Manual de buenas prácticas de post-cosecha para flor de corte y follajes asociados, Versión 2, AFIF, Asocolflores, 2010.

Ferrin, C., Magdalena, X., Loaiza, H., López, S., \& Henao, S. (2014). Sistema de extracción automática de parámetros morfológicos de la huella plantar mediante técnicas de visión por computador en un sistema embebido. Revista Colombiana de Tecnologías de Avanzada, 1, 80-86.
Katupitiya, J., Eaton, R., Yaqub, T. (2007) Systems engineering approach to agricultural automation: New developments. Proceedings of the 1st Annual 2007 IEEE Systems Conference, 298-304. http://doi.org/10.1109/SYSTEMS.2007.374688

Monroy N., Pérez I., Cure J. R. Modelo de predicción y manejo de cultivos de rosas. Universidad de los Andes, Facultad de Ingeniería, Rev 15- Art 3. 2002.

Pardo, C., Gutierrez, E., Jiménez, F., Sosa, L., \& Martínez, L. (2013). Diseño e implementación de un prototipo de sistema de identificación y clasificación de piezas por tratamiento digital de imágenes con acceso a ethernet y comunicación inalámbrica. Revista Colombiana de Tecnologías de Avanzada, 2, 49-58.

Sistler, F. E., Bldg, E. B. D., \& Rouge, B. (n.d.). Grading Agricultural Products with Machine VISION. Louisiana State University Agricultural Center, 255-261.

Siraj, F., Ekhsan, H. M., \& Zulkifli, A. N. (2014). Flower Image Classification Modeling Using Neural Network, 81-86.

Zawbaa, H. M., Abbass, M., \& Basha, S. H. (2014). An Automatic Flower Classification Approach Using Machine Learning Algorithms. Icacci, 895-901. http://doi.org/10.1109/ICACCI.2014.6968612

Zou J., Nagy G. Evaluation of Model-Based Interactive Flower Recognition. Renssealer Polytechnic Institute, DocLab, ECSE department, 2004.

\section{SITIOS WEB}

QtSerialPort, consultado el 11 Diciembre 2013. http://qt-project.org/wiki/QtSerialPort.

Install Open CV, consultado el 11 Diciembre 2013. http://docs.opencv.org/doc/tutorials/introductio n/linux_install/linux_install.ht ml. 